\title{
O SOBRE-SINAL E A RESPOSTA INVERSA EM SISTEMAS DE CONTROLE LINEARES CONTÍNUOS NO TEMPO
}

\author{
Célia Aparecida dos Reis \\ Universidade Estadual Paulista - \\ UNESP, Campus de Ilha Solteira, \\ Departamento de Matemática, \\ Caixa Postal 31, CEP 15385-000, \\ Ilha Solteira, SP, Brasil \\ célia@mat.feis.unesp.br
}

\author{
Ali Messaoudi \\ Universidade Estadual Paulista - \\ UNESP, IBILCE, Departamento de \\ Matemática, São José do Rio Preto, \\ SP, Brasil. \\ messaoud@ibilce.unesp.br
}

\author{
Neusa A. P. Silva \\ Universidade Estadual Paulista \\ - UNESP, Campus de Ilha \\ Solteira, Departamento de \\ Matemática, Caixa Postal 31, \\ CEP 15385-000, Ilha Solteira, \\ SP, Brasil \\ neusa@mat.feis.unesp.br
}

\begin{abstract}
This paper deals with the problem of extrema, which may occur, in the step-response of a linear system with real zeros and poles. Necessary and sufficient conditions for extrema, overshoot and undershoot in the step-response of $n$ $\geq 2$ order continuous time transfer functions with distinct poles and one real zero are given. The results are expressed in terms of the locations of poles and zeros of the corresponding transfer-function.
\end{abstract}

KEYWORDS: Extrema, overshoot, undershoot, poles, zero.

\section{RESUMO}

Este trabalho trata da determinação dos extremos que podem ocorrer na resposta a uma entrada degrau unitário de sistemas lineares contínuos no tempo, com pólos e zeros reais. Apresentam-se condições necessárias e suficientes para avaliar extremos, sobre-sinal e resposta inversa na resposta a uma entrada degrau unitário de sistemas de ordem $n \geq 2$ com um zero real e pólos reais distintos. Todos os resultados são apresentados em termos das localizações de pólos e zeros da correspondente função de transferência.

PALAVRAS-CHAVE: Extremos, sobre-sinal, resposta inversa, pólos, zero.

Artigo Submetido em 28/05/03

1a. Revisão em 20/10/03

2a. Revisão em 03/05/04

3 a Revisão em 19/010/04

Aceito sob recomendação do Ed. Assoc.Prof. José R. C. Piqueira

\section{INTRODUÇÃO}

O conhecimento das características da resposta a degrau é importante em engenharia de controle. De fato, existem alguns problemas tais como de controle do eixo de máquinas ferramentas e problemas nos quais um robô necessita seguir uma trajetória pré-definida, em que a resposta a degrau não pode apresentar extremos (El-Khoury et alii, 1993; Howell, 1997; Rachid, 1995; Leon de la Barra, 1994). Dessa forma, o estudo de condições que permitam avaliar extremos, sobre-sinal e resposta inversa na resposta a degrau, é de grande importância na teoria de controle.

Muitas contribuições teóricas recentes têm sido feitas no sentido de clarificar a influência dos zeros e das localizações de pólos e zeros da planta na parte transiente da resposta a degrau. El-Khoury et alii (1993) apresenta um limitante superior para o número de extremos na resposta a degrau de um sistema linear estável, complementando os resultados existentes na literatura para limitantes inferiores. Rachid (1995) fornece uma condição suficiente para avaliar extremos, usando a configuração pólos-zeros da correspondente função de transferência. Tal resultado fornece em que condições a resposta a degrau não apresenta sobre-sinal. Howell (1997) apresenta uma nova classe de respostas a degrau sem extremos, as quais podem ser usadas ao invés de um modelo multi-exponencial, que pode apresentar o efeito do sobre-sinal ou de resposta inversa. Goodwin et alii (1999) considera o efeito de zeros estáveis próximos ao eixo jw para sistemas SISO. Mostra-se que a 
presença de tais zeros, juntamente com limitantes superiores para o sobre-sinal permissível do sinal de saída, fornece um limitante superior para o tempo de acomodação do sistema de malha fechada. León de la Barra (1994) mostra que a resposta a degrau de um sistema contínuo no tempo, assintoticamente estável, estritamente próprio e de fase não mínima, com $m_{l}$ zeros reais no semiplano aberto direito e sem pólos e zeros complexos conjugados, exibe resposta inversa exatamente $\operatorname{int}\left[\left(m_{1}+1\right) / 2\right]$ vezes, sendo que int [ ] denota a parte inteira. Lin e Fang (1997) apresentam uma condição necessária e suficiente para um sistema linear SISO de terceira ordem, com pólos reais, ter resposta a degrau sem sobre-sinal ou ser esta resposta monótona não decrescente. No caso de pólos complexos, uma condição suficiente e duas condições necessárias são obtidas. Observa-se que todas as condições encontradas são dadas em termos dos coeficientes do numerador da função de transferência. Moore (1990) apresenta uma técnica para a escolha de localizações de zeros para a obtenção de sobresinal mínimo.

Apesar de bastante valiosas, tais contribuições ainda não oferecem um quadro claro de como e quais variações extensas nas localizações de pólos e zeros podem influenciar o sobre-sinal e a resposta inversa. Por exemplo, o problema de se determinar o número exato de extremos da resposta a degrau permanece em aberto (El-Khoury et alii, 1993) e, mesmo determinando os extremos da resposta a degrau, não existe uma técnica que permita classificá-los, ou seja, faltam condições necessárias e suficientes para a prova da existência de extremos, sobre-sinal e resposta inversa.

Como contribuição dos autores (Reis e Silva, 2001, 2002; Reis et alii, 2004, 2004-a, Silva e Reis, 2001), foram obtidas condições necessárias e suficientes para a existência de extremos, sobre-sinal e resposta inversa em sistemas de segunda e terceira ordem, com pólos e zeros reais. Portanto, neste trabalho, será apresentada uma generalização para determinação de extremos, sobre-sinal e resposta inversa em sistemas de controle lineares contínuos no tempo e de ordem $n$, com um zero real.

Acreditamos que as caracterizações obtidas são de importância na teoria de controle já que permitem uma visão mais esclarecida das condições que possibilitem avaliar extremos, sobre-sinal e resposta inversa em sistemas lineares, além de clarificar um pouco mais a influência dos zeros e localizações de pólos e zeros da planta na parte transiente da resposta a degrau.

Este trabalho está organizado como segue. Na Seção 2, apresentam-se as considerações iniciais sobre o trabalho. Na Seção 3, apresentam-se os resultados principais relativos a extremos, sobre-sinal e resposta inversa. $\mathrm{Na}$
Seção 4, as conclusões do trabalho. No Apêndice, encontram-se as provas dos resultados auxiliares.

\subsection{CONSIDERAÇÕES INICIAIS}

Considera-se o sistema de controle linear contínuo no tempo, monovariável e estável descrito pela função de transferência:

$$
G(s)=\frac{(T s+1)}{\prod_{j=1}^{n}\left(\tau_{j} s+1\right)} \text { ou } G(s)=\frac{(-T s+1)}{\prod_{j=1}^{n}\left(\tau_{j} s+1\right)}
$$

sendo que $n \geq 2$, e:

$T$ é uma constante real estritamente positiva;

- $\tau_{j}>0, j=1,2, \ldots, n$ são as constantes de tempo do sistema;

- $z=-1 / T$, é o zero de $\mathrm{G}(\mathrm{s})$ se $\mathrm{z}<0$ ou $z=1 / T$ se $\mathrm{z}>0$; - $\lambda_{j}=-1 / \tau_{j}, \mathrm{j}=1,2, \ldots$, n são os pólos de $\mathrm{G}(\mathrm{s})$;

- $\lambda_{j} \neq \lambda_{i}, \lambda_{j} \neq z$ para todo $\mathrm{i}, \mathrm{j}=1,2, \ldots, \mathrm{n}$.

Considera-se, ainda, sem perda de generalidade, que:

$$
\lambda_{1}<\ldots<\lambda_{n-1}<\lambda_{n}<0 \text { e } t \in[0,+\infty)
$$

Observa-se que $G(s)$ pode ser escrita, na forma de pólos e zeros, do seguinte modo:

$$
G(s)=\frac{(-1)^{n+1} \prod_{j=1}^{n} \lambda_{j}}{z} \frac{(s-z)}{\prod_{j=1}^{n}\left(s-\lambda_{j}\right)} .
$$

O lema, apresentado a seguir, fornece a resposta a uma entrada degrau unitário para a classe de sistemas cuja função de transferência tem a forma dada por (3).

LEMA 01: A resposta a degrau da classe de sistemas de controle linear, cuja função de transferência é dada por (2.3) e tem condições iniciais $y(0)=0, y^{\prime}(0)=0, \ldots$, $y^{(n-1)}(0)=k$ sendo que $k=(-1)^{n+1} \frac{\prod_{i=1}^{n} \lambda_{i}}{z}$, tem a forma:

$$
y(t)=1+\sum_{i=1}^{n} c_{i} e^{\lambda_{i} t}
$$

na qual: 
(i). $c_{1}=\frac{\lambda_{2} \cdots \lambda_{n}}{z}\left(\lambda_{1}-z\right) \frac{\prod_{2 \leq i<j \leq n}\left(\lambda_{j}-\lambda_{i}\right)}{\prod_{1 \leq i<j \leq n}\left(\lambda_{j}-\lambda_{i}\right)}$;

(ii). $c_{k}=(-1)^{k+1} \frac{\prod_{i=1}^{n} \lambda_{i}}{\lambda_{k} z}\left(\lambda_{k}-z\right) \frac{\prod_{\substack{1 \leq i<j \leq n \\ \mathrm{i}, \mathrm{j} \neq k}}\left(\lambda_{j}-\lambda_{i}\right)}{\prod_{1 \leq i<j \leq n}\left(\lambda_{j}-\lambda_{i}\right)}$, . $\forall 2 \leq \mathrm{k} \leq \mathrm{n}$

PROVA: Ver Apêndice.

O corolário, apresentado a seguir, é uma conseqüência do Lema 01.

COROLÁRIO 01: Sob as hipóteses do Lema 01 e sendo $n$ a ordem do sistema dado por (3), então:

(a) Se $\lambda_{n}<z<0$ tem-se que:

Se $n$ é par então $c_{1}<0, c_{2}>0, c_{3}<0, \ldots ., c_{n}>0$;

Se $n$ é ímpar então $c_{1}>0, c_{2}<0, c_{3}>0, \ldots ., c_{n}>0$.

Se $\lambda_{n}<0<z$ tem-se que:

Se $n$ é par então $c_{1}>0, c_{2}<0, c_{3}>0, \ldots ., c_{n},<0$;

Se $n$ é ímpar então $c_{1}<0, c_{2}>0, c_{3}<0, \ldots ., c_{n}<0$.

PROVA: Ver Apêndice.

\subsection{DETERMINAÇÃO DE SOBRE-SINAL E DE RESPOSTA INVERSA}

Tem-se, agora, o seguinte resultado principal, o qual fornece uma condição necessária e suficiente para a existência de pontos críticos na resposta a uma entrada degrau unitário de (1) ou (3) e a classificação dos mesmos.

TEOREMA 01: Considera-se o sistema de controle linear contínuo no tempo, monovariável, com $n$ pólos reais distintos e com um zero real, estável descrito pela função de transferência (3), cuja resposta a uma entrada degrau unitário $y(t)$ tem a forma (4) - (6). Têm-se, então, os seguintes resultados:

(a) $y(t)$ não apresenta extremos se e somente se $z<\lambda_{n}$;

(b) $y(t)$ apresenta um ponto de máximo se e somente se $\lambda_{n}$ $<z<0$;

(c) $y(t)$ apresenta um ponto de mínimo se e somente se $\lambda_{n}$ $<0<z$
Uma observação a ser feita é que o item (a) pode ser deduzido do trabalho de Rachid (1995). Neste trabalho, será efetuada uma prova elementar.

Para a prova do Teorema 01, necessita-se de algumas considerações preliminares as quais são apresentadas a seguir. Inicialmente, de (4), tem-se que:

$$
y^{\prime}(t)=\sum_{i=1}^{n} c_{i} \lambda_{i} e^{\lambda_{i} t}
$$

Em (7), fazendo:

$$
c_{i}^{(l)}=c_{i} \lambda_{i}, \quad d_{i}^{(I)}=\frac{c_{i}^{(l)}}{c_{l}^{(l)}} \quad \text { e } \quad \lambda_{i}^{(I)}=\lambda_{i}-\lambda_{l},
$$

obtém-se:

$$
y^{\prime}(t)=0 \Leftrightarrow 1+\sum_{i=2}^{n} d_{i}^{(I)} e^{\lambda_{i}^{(l)} t}=0 .
$$

Defina,

$$
f_{1}(t)=1+\sum_{i=2}^{n} d_{i}^{(l)} e^{\lambda_{i}^{(I)} t}
$$

Observa-se de (9) que $f_{l}(0)=0$, pois $y^{\prime}(0)=0$. Além disso, para a análise de $f_{l}(t)$, torna-se necessário a análise do sinal de $d_{i}^{(l)}$. Apresenta-se, a seguir, a análise do caso $d_{n}^{(l)}>0$. O caso $d_{n}^{(1)}<0$ é feito de forma análoga.

Suponha que $d_{n}^{(l)}>0$. De (9) conclui-se que $f_{l}(0)=\frac{y^{\prime}(0)}{c_{l}^{(1)}}$ $=0$ e como $d_{n}^{(l)}>0, \lim _{t \rightarrow+\infty} f_{l}(t)=+\infty$. Assim, ou $f_{l}(t)$ é uma função positiva com valor inicial nulo ou $f_{l}(t)$ é uma função com valores positivos e negativos, de valor inicial nulo, cortando o eixo real em pelo menos um ponto. Portanto a figura 01, dada a seguir, mostra três possibilidades para o gráfico de $f_{l}(t)$ satisfazendo $f_{l}(0)=0$ e $\lim _{t \rightarrow+\infty} f_{l}(t)=+\infty$.

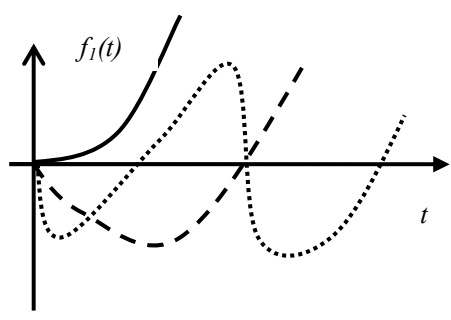

Figura 01: Possíveis formas para o gráfico de $f_{l}(t)$. 
Então, existe um número real positivo $t_{o}^{*}$ tal que $y^{\prime}\left(t_{o}^{*}\right)=$ 0 , se e somente se existe $t_{o}$ pertencente a $(0,+\infty)$ tal que $f_{1}^{\prime}\left(t_{o}\right)=0$. Mas de (9),

$$
f_{1}^{\prime}(t)=\sum_{i=2}^{n} d_{i}^{(l)} \lambda_{i}^{(l)} e^{\lambda_{i}^{(l)} t}
$$

Em (10), fazendo:

$$
c_{i}^{(2)}=d_{i}^{(1)} \lambda_{i}^{(1)}, d_{i}^{(2)}=\frac{c_{i}^{(2)}}{c_{2}^{(2)}} \quad \text { e } \quad \lambda_{i}^{(2)}=\lambda_{i}^{(1)}-\lambda_{2}^{(1)}
$$

obtém-se que:

$$
f_{l}^{\prime}(t)=0 \Leftrightarrow 1+\sum_{i=3}^{n} d_{i}^{(2)} e^{\lambda_{i}^{(2)} t}=0
$$

Defina

$$
f_{2}(t)=1+\sum_{i=3}^{n} d_{i}^{(2)} e^{\lambda_{i}^{(2)} t}
$$

De (11) observa-se que:

(i). $f_{2}(0)=0$ e $\lim _{t \rightarrow+\infty} f_{2}(t)=+\infty$ se $d_{n}^{(2)}>0$ ou

(ii). $\lim _{t \rightarrow+\infty} f_{2}(t)=-\infty$ se $d_{n}^{(2)}<0$.

Em ambos os casos, existe um número real $t_{o}^{*}$ tal que $y^{\prime}\left(t_{o}^{*}\right)=0$ se e somente se existe $t_{o}$ pertencente a $(0,+\infty)$ tal que $f_{2}^{\prime}\left(t_{o}\right)=0$. Defina

$$
f_{k}(t)=1+\sum_{i=k+1}^{n} d_{i}^{(k)} e^{\lambda_{i}^{(k)} t} \quad \forall 2 \leq k \leq n-2
$$

sendo:

(i). $\quad c_{i}^{(k)}=d_{i}^{(k-1)} \lambda_{i}^{(k-1)}$;

(ii). $\quad d_{i}^{(k)}=\frac{c_{i}^{(k)}}{c_{k}^{(k)}}$;

(iii). $\lambda_{i}^{(k)}=\lambda_{i}^{(k-1)}-\lambda_{k}^{(k-1)}=\lambda_{i}-\lambda_{k}$.

Continuando com o processo anterior, tem-se que existe um número real $t_{o}^{*}$ tal que $y^{\prime}\left(t_{o}^{*}\right)=0$ se e somente se existe $t_{o}$ pertencente a $(0,+\infty)$ tal que $f_{n-2}^{\prime}\left(t_{0}\right)=0$.
Decorrem, então, os lemas seguintes que fornecem uma condição necessária e suficiente para a existência de extremos em $y(t)$.

LEMA 02: Sob as hipóteses do Teorema $01 y(t)$ tem um extremo $t_{o}^{*}$ se e somente se $-\frac{d_{n-1}^{(n-2)} \lambda_{n-1}^{(n-2)}}{d_{n}^{(n-2)} \lambda_{n}^{(n-2)}}>1$.

PROVA: Ver Apêndice.

LEMA 03: Sob as hipóteses do Teorema 01, tem-se que $-\frac{d_{n-1}^{(n-2)} \lambda_{n-1}^{(n-2)}}{d_{n}^{(n-2)} \lambda_{n}^{(n-2)}}>1$ se e somente se $\lambda_{n}<z$.

PROVA: Ver Apêndice.

Apresenta-se, a seguir, a prova do Teorema 01.

PROVA DO TEOREMA 01: Pelo Lema 02, $y(t)$ tem um extremo $t_{o}^{*}$ se e somente se $-\frac{d_{n-1}^{(n-2)} \lambda_{n-1}^{(n-2)}}{d_{n}^{(n-2)} \lambda_{n}^{(n-2)}}>1$. Pelo Lema 03, $-\frac{d_{n-1}^{(n-2)}}{d_{n}^{(n-2)}} \frac{\lambda_{n-1}^{(n-2)}}{\lambda_{n}^{(n-2)}}>1$ se e somente se $\lambda_{n}<z$. Dessa forma, o item (a) está provado. Para a prova de (b), suponha que:

$$
\lambda_{n}<z<0 .
$$

De (9), (11) e (12) conclui-se que:

$$
\begin{aligned}
& y^{\prime}(t)=c_{1}^{(1)} e^{\lambda_{1} t} f_{1}(t) \\
& f_{1}^{\prime}(t)=c_{2}^{(2)} e^{\lambda_{2}^{(I)} t} f_{2}(t)
\end{aligned}
$$

$$
f_{n-2}^{\prime}(t)=c_{n-1}^{(n-1)} e^{\lambda_{n-1}^{(n)}} f_{n-1}(t)
$$

De (14), tem-se que:

$$
\begin{aligned}
& \sin \operatorname{al}\left(y^{\prime}(t)\right)=\sin \operatorname{al}\left(c_{1}^{(1)} f_{1}(t)\right) \\
& \sin \operatorname{al}\left(f_{1}^{\prime}(t)\right)=\sin \operatorname{al}\left(c_{2}^{(2)} f_{2}(t)\right)
\end{aligned}
$$

$\operatorname{sinal}\left(f_{n-2}^{\prime}(t)\right)=\sin \operatorname{al}\left(c_{n-1}^{(n-1)} f_{n-1}(t)\right)$

Agora, de (12), decorre que: 


$$
f_{k}(t)=1+\sum_{i=k+1}^{n} d_{i}^{(k)} e^{\lambda_{i}^{(k)} t}
$$

De (5) e (8) mostra-se que:

$$
. c_{1}^{(I)}=c_{1} \lambda_{1}=\frac{\lambda_{1} \lambda_{2} \cdots \lambda_{n}}{z}\left(\lambda_{1}-z\right) \frac{\prod_{2 \leq i<j \leq n}\left(\lambda_{j}-\lambda_{i}\right)}{\prod_{1 \leq i<j \leq n}\left(\lambda_{j}-\lambda_{i}\right)} .
$$

Logo, como $\lambda_{1}<\lambda_{2}<\cdots<\lambda_{n}<z<0$,

$$
\sin a l\left(c_{1}^{(l)}\right)=(-1)^{n} \sin a l\left(\frac{\lambda_{1}-z}{z}\right)=(-1)^{n} .
$$

Agora, de (8) e (13) - (i) e (iii), tem-se que:

$$
c_{2}^{(2)}=d_{2}^{(I)} \lambda_{2}^{(I)}=\frac{c_{2}^{(1)}}{c_{1}^{(I)}}\left(\lambda_{2}-\lambda_{1}\right)=\frac{c_{2}}{c_{1}} \frac{\lambda_{2}}{\lambda_{1}}\left(\lambda_{2}-\lambda_{1}\right) .
$$

Portanto:

$$
\operatorname{sinal}\left(c_{2}^{(2)}\right)=\sin a l\left(\frac{c_{2}}{c_{1}}\right)<0 .
$$

Mas de (13),

$$
\begin{aligned}
c_{3}^{(3)}= & \frac{c_{3}^{(2)}}{c_{2}^{(2)}} \lambda_{3}^{(2)}=\frac{d_{3}^{(1)}}{d_{2}^{(1)}} \frac{\lambda_{3}^{(1)}}{\lambda_{2}^{(1)}} \lambda_{3}^{(2)}= \\
& \frac{c_{3} \lambda_{3}}{c_{2} \lambda_{2}} \frac{\left(\lambda_{3}-\lambda_{1}\right)}{\left(\lambda_{2}-\lambda_{1}\right)}\left(\lambda_{3}-\lambda_{2}\right) .
\end{aligned}
$$

Então, observa-se que:

$$
\sin a l\left(c_{3}^{(3)}\right)=\sin a l\left(\frac{c_{3}}{c_{2}}\right)<0 .
$$

De forma análoga obtém-se que:

$$
\begin{gathered}
\operatorname{sinal}\left(c_{4}^{(4)}\right)=\operatorname{sinal}\left(\frac{c_{4}}{c_{3}}\right)<0 \\
\ldots \ldots \\
\operatorname{sinal}\left(c_{n-1}^{(n-1)}\right)=\operatorname{sinal}\left(\frac{c_{n-1}}{c_{n-2}}\right)<0 .
\end{gathered}
$$

Pode ocorrer que $n$ seja um número par ou ímpar. Inicialmente apresenta-se a análise para $n$ par.
Caso 01: $n$ é um número par.

Resulta do Corolário 01 e de (1) - (13), que:

$$
d_{n}^{(1)}=\frac{c_{n}^{(1)}}{c_{1}^{(1)}}=\frac{c_{n}}{c_{1}} \frac{\lambda_{n}}{\lambda_{1}}<0 .
$$

Além disso,

$$
d_{n}^{(2)}=\frac{c_{n}^{(2)}}{c_{2}^{(2)}}=\frac{d_{n}^{(1)}}{d_{2}^{(1)}} \frac{\lambda_{n}^{(1)}}{\lambda_{2}^{(1)}}>0
$$

pois $d_{n}^{(1)}<0$ e $d_{2}^{(1)}<0$. Continuando com esse processo, conclui-se que:

$$
d_{n}^{(3)}<0, d_{n}^{(4)}>0, \ldots, d_{n}^{(n-1)}<0 .
$$

Agora, como:

$$
f_{n-1}(t)=1+d_{n}^{(n-1)} e^{\lambda_{n}^{(n-1)} t},
$$

tem-se que $\lim _{t \rightarrow+\infty} f_{n-l}(t)=-\infty$ e $f_{n-1}(0)>0$.

Então, o gráfico de $f_{n-1}$ tem a forma dada pela figura 02, a seguir:

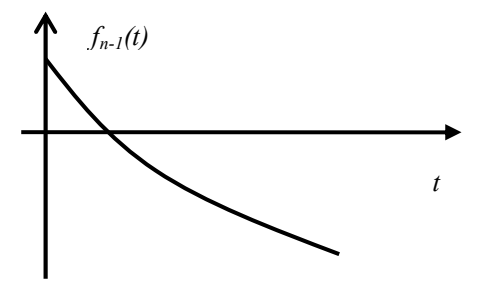

Figura 02: Forma do gráfico de $f_{n-1}(t)$.

Agora, como:

$$
\begin{gathered}
\operatorname{sinal}\left(f_{n-2}^{\prime}(t)\right)=\operatorname{sinal}\left(c_{n-1}^{(n-1)} f_{n-1}(t)\right) \\
=-\operatorname{sinal}\left(f_{n-1}\right),
\end{gathered}
$$

o gráfico de $f_{n-2}$ tem a forma dada na figura 03 , a seguir:

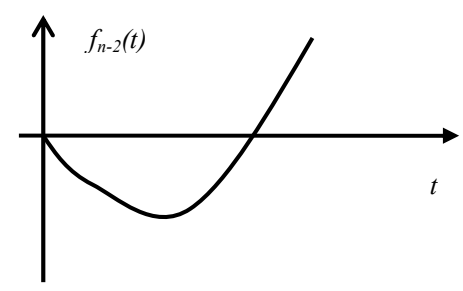

Figura 03: Forma do gráfico de $f_{n-2}(t)$. 
Assim, $\operatorname{sinal}\left(f_{n-3}^{\prime}\right)=-\operatorname{sinal}\left(f_{n-2}\right)$. Então o gráfico de $f_{n-3}(t)$ tem a forma:

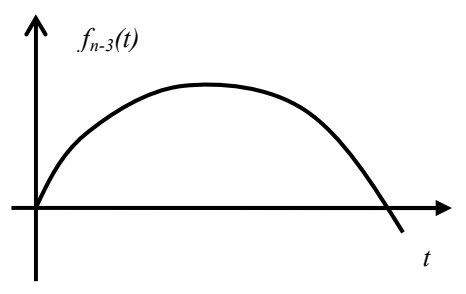

Figura 04: Forma do gráfico de $f_{n-3}(t)$.

Continuando com este processo, como $\sin a l\left(c_{1}^{(1)}\right)=(-1)^{n}$ $=1$, conclui-se que o $\sin a l\left(y^{\prime}\right)=\operatorname{sinal}\left(f_{1}\right)$ e a forma do gráfico de $f_{l}(t)$ é do tipo:

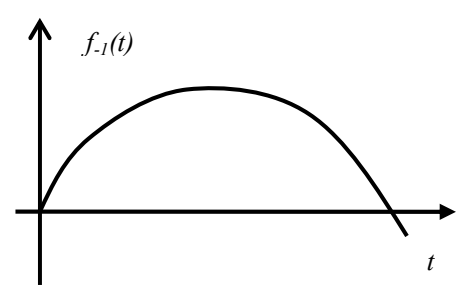

Figura 05: Forma do gráfico de $f_{l}(t)$.

Então, o gráfico de y(t) tem a forma dada a seguir:

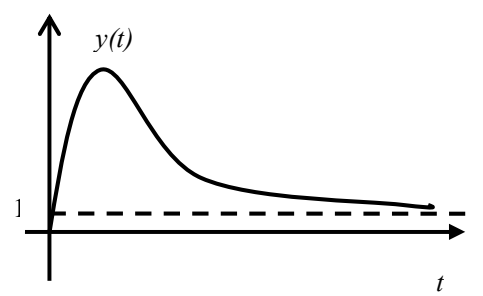

Figura 06: Forma do gráfico de $y(t)$.

Portanto, $y(t)$ tem um máximo.

Caso 02: $n$ é um número real ímpar:

Segue do Corolário 01 que:

$$
d_{n}^{(1)}=\frac{c_{n}^{(1)}}{c_{1}^{(1)}}=\frac{c_{n}}{c_{1}} \frac{\lambda_{n}}{\lambda_{1}}>0 .
$$

De forma análoga:

$$
d_{n}^{(2)}<0, d_{n}^{(3)}>0, \ldots, d_{n}^{(n-1)}<0 .
$$

Logo, conclui-se que:

$$
\lim _{t \rightarrow+\infty} f_{n-1}(t)=-\infty \text { e } f_{n-1}(0)>0 .
$$

Então o gráfico de $f_{n-1}(t)$ tem a forma:

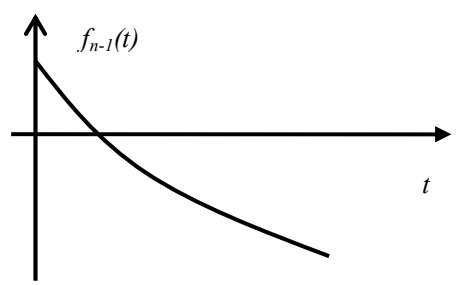

Figura 07: Forma do gráfico de $f_{n-l}(t)$.

Agora, como:

$$
\begin{gathered}
\operatorname{sinal}\left(f_{n-2}^{\prime}(t)\right)=\operatorname{sinal}\left(c_{n-1}^{(n-1)} f_{n-1}(t)\right) \\
=-\operatorname{sinal}\left(f_{n-1}\right),
\end{gathered}
$$

o gráfico de $f_{n-2}$ tem a forma dada pela figura 08 , a seguir:

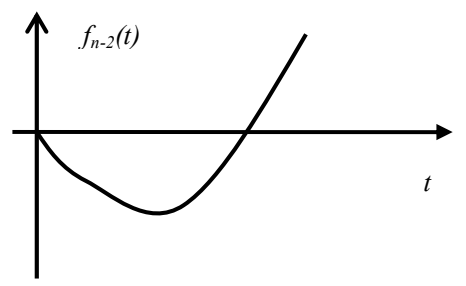

Figura 08: Forma do gráfico de $f_{n-2}(t)$.

Por outro lado, $\operatorname{sinal}\left(f_{n-3}^{\prime}\right)=-\operatorname{sinal}\left(f_{n-2}\right)$. Então o gráfico de $f_{n-3}(t)$ tem a forma dada pela figura 09.

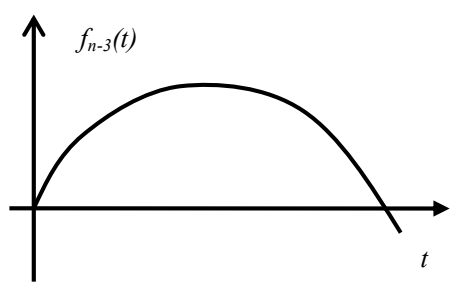

Figura 09: Forma do gráfico de $f_{n-3}(t)$.

Continuando com este processo, obtém-se o gráfico de $f_{l}(t)$ dado na figura 10.

Agora, como:

$$
\sin \operatorname{al}\left(y^{\prime}\right)=(-1)^{n} \sin \operatorname{al}\left(f_{1}\right)=-\sin \operatorname{al}\left(f_{1}\right),
$$


o gráfico de $y(t)$ tem a forma dada na figura 11, a seguir:

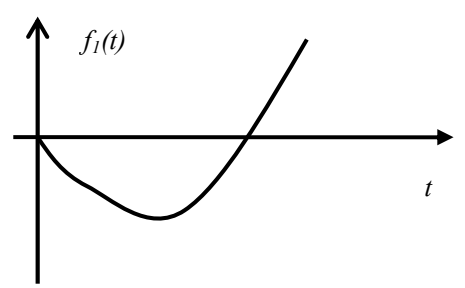

Figura 10: Forma do gráfico de $f_{l}(t)$.

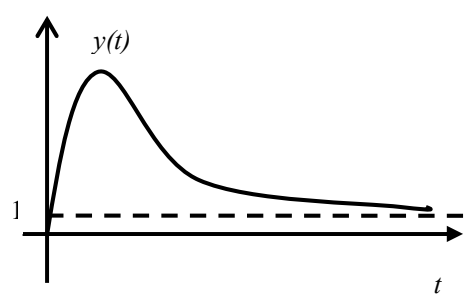

Figura 11: Forma do gráfico de $y(t)$.

Portanto, $y(t)$ tem um máximo. O caso $z>0$ se faz de forma análoga e o teorema está provado.

O corolário, a seguir, apresenta condições necessárias e suficientes para a existência de sobre-sinal e resposta inversa em sistemas de ordem $\mathrm{n}$ com um zero real.

COROLÁRIO 02: Considera-se o sistema de controle linear contínuo no tempo, monovariável, com n pólos reais distintos e com um zero real, estável descrito pela função de transferência (1) e (3), cuja resposta a uma entrada degrau unitário tem a forma (4) - (6). Têm-se, então, os seguintes resultados:

(a) $y(t)$ apresenta sobre-sinal se e somente se $\lambda_{n}<z<0$;

(b) $y(t)$ apresenta resposta inversa se e somente se $\lambda_{n}<0<z$;

Vale destacar que no caso $z>0$, foi provado a existência de um ponto de mínimo na resposta a degrau unitário, além da existência de resposta inversa. Leon de La Barra (1994) também mostra, neste caso, a existência de resposta inversa. Além da prova da existência de extremos e classificação dos mesmos, uma outra contribuição do Teorema 01 está na sua demonstração, visto que a mesma apresenta um caminho que permitirá, possivelmente, a análise de sistemas de ordem $n$, apresentando $m$ zeros, com $m \leq n$.

Para o caso em que $n=3$, observa-se que $y(t)$, dada por (1) ou (3), apresenta sobre-sinal se e somente $\lambda_{1}<\lambda_{2}<\lambda_{3}<z<0$. Nota-se, neste caso, que tal resultado é similar aos resultados obtidos no Lema 02 e Teorema 03 de Reis e Silva (2001), para sistemas de segunda ordem.

EXEMPLO 01: Considere o sistema de controle linear cuja função de transferência seja dada por:

$$
G(s)=\frac{4 s+1}{\left(\frac{1}{4} s+1\right)\left(\frac{1}{3} s+1\right)(s+1)} .
$$

Observa-se que o sistema possui um zero $z=-1 / 4$ e os pólos $\lambda_{3}=-1, \lambda_{2}=-3$ e $\lambda_{1}=-4$. A figura 12, dada a seguir, mostra o gráfico da resposta a uma entrada degrau unitário, para este sistema.

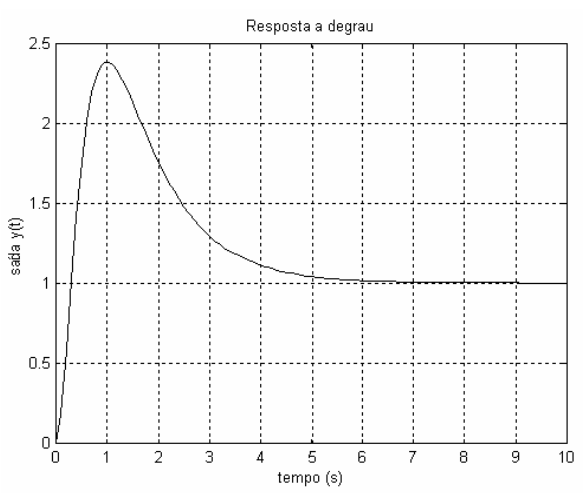

Figura 12: Resposta a degrau unitário de $G(s)$ em (15).

De acordo com o Teorema 01 e da figura 12, $y(t)$ apresenta sobre-sinal superior a $100 \%$.

Exemplo 02: Considere o sistema cuja função de transferência seja dada por:

$$
G(s)=\frac{\left(\frac{-1}{2} s+1\right)}{(s+1)\left(\frac{1}{2} s+1\right)\left(\frac{1}{3} s+1\right)} .
$$

Observa-se que $G(s)$ apresenta um zero real z $=2$ e pólos $\lambda_{3}=-1, \lambda_{2}=-2$ e $\lambda_{1}=-3$. A figura 13 , a seguir, mostra $o$ gráfico da saída $y(t)$. Nota-se, neste caso, a ocorrência de resposta inversa inicial, comportamento este, exclusivamente devido à presença do zero real positivo.

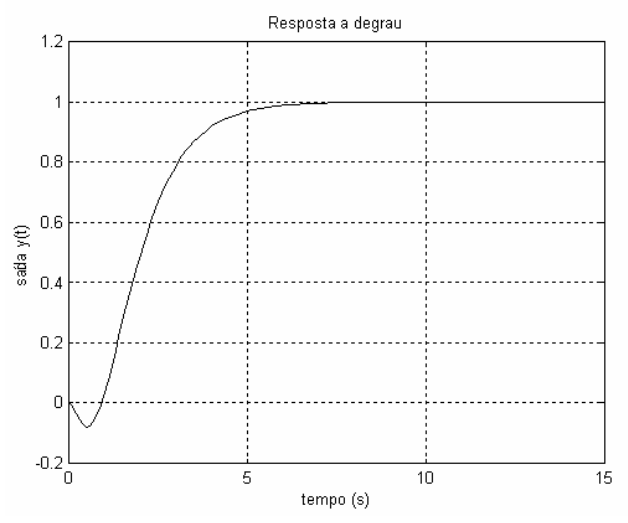

Figura 13: Resposta a degrau unitário de $G(s)$ em (16). 


\section{CONSIDERAÇÕES FINAIS}

Foram obtidas, neste trabalho, condições necessárias e suficientes para a determinação e classificação dos extremos da resposta a uma entrada degrau unitário, em sistemas de ordem $n$ com pólos reais distintos e com um zero real, os Lemas 02, 03 e o Teorema 01. Como conseqüências diretas do Teorema 01 foram obtidas condições necessárias e suficientes para a ocorrência de sobre-sinal e resposta inversa nesta classe de sistemas, em função das posições relativas dos pólos e do zero, o Corolário 02.

Observa-se que tais resultados coincidem com os resultados obtidos para sistemas de segunda ordem com pólos reais e um zero real (Reis e Silva, 2001; Reis et alii, 2002).

Acredita-se que, com os resultados obtidos neste trabalho, foi dado um passo importante para um melhor entendimento da influência das posições relativas de pólos e zeros para ocorrência de extremos, sobre-sinal e resposta inversa em sistemas de controle lineares e contínuos no tempo.

\section{AGRADECIMENTOS}

Agradecemos a FAPESP pelo apoio via o projeto de pesquisa 98/06085-6.

\section{REFERÊNCIAS BIBLIOGRÁFICAS}

Dorf R. C. and R. H. Bishop (1995). Modern Control Systems, 7 ed. Addison-Wesley, New York.

El-Khoury, M., O. D. Crisalle and R. Longchamp (1993). Influence of Zero Locations on the Number of Stepresponse Extrema. Automatica, vol. 29, nº 6, pp. 15711574.

Franklin, G. F., J. D. Powell and A. Emani-Naeini (1991). A. Feedback Control of Dynamic Systems, 3ed. Addison-Wesley, New York.

Goodwin, G. C., A. R. Woodyatt, R. H. Diddleton and J. Shim (1999). Fundamental Limitations due to jw-axis zeros in SISO Systems. Automatica, vol. 35, pp. 857 863.

Howell, J. R. (1996). Comment Regarding "On undershoot in SISO Systems”. IEEE Trans. Automat. Control, vol. 41, n⿳ํㅡㄹ 12, pp. 1845 - 1846.

Howell, J. R. (1997). Some classes of Step-response Model Without Extrema. Automatica, vol. 33, no 7, pp. 1427 1428.

León de la Barra, B.A. (1994). On undershoot in SISO systems. IEEE Trans. Automat. Control, vol. 39, nº 3, pp. 578-581.
León de la Barra, B.A. and M. A. Fernández (1994-a). Transient Properties of Type $m$ Continuous Time Scalar Systems. Automática, vol. 30, nº 9, pp. 14951496.

Lin, S. and C. Fang (1997). "Nonovershooting and Monotone Nondecreasing Step Response of a ThirdOrder SISO Linear System”. IEEE Trans. Automat. Control, vol. 42, nº 9, pp. 1299-1303.

MATLAB The Language of Technical Computing, Version 5, 1997.

Moore, K. L and S. P. Bhattacharyya (1990). "A Technique for Choose Zero Locations for Minimal Overshoot”. IEEE Trans. Automat. Control, vol. 35, nํ 5, pp. 577580.

Ogata, K. (1998). Engenharia de Controle Moderno. Prentice-Hall do Brasil, Rio de Janeiro.

Rachid, A. (1995). Some conditions on zeros to avoid stepresponse extrema. IEEE Trans. Automat. Control, vol. 40, $\mathrm{n}^{-0}$ 8, pp. 1501-1503.

Reis, C. A. and N. A. P. Silva (2001). Condições Necessárias e Suficientes Para a Existência de Overshoot em Sistemas Lineares Contínuos e de Segunda Ordem. Anais da $1^{a}$ Escola Brasileira de Aplicações em Dinâmica e Controle, São Carlos, S. P., pp.176-179.

Reis, C. A., N. A. P. Silva and M. C. M. Teixeira, (2002). O Overshoot e o Undershoot em Sistemas de Controle Lineares Contínuos no Tempo: Um Tutorial. Segundo Congresso Nacional de Engenharia Mecânica (CONEM 2002), João Pessoa, PB.

Reis, C. A., (2002-a). O Tempo de Subida em Sistemas de Controle Lineares e Contínuos no Tempo. Anais do I Congresso de Dinâmica, Controle e Aplicações (DINCOM 2002), São José do Rio Preto, S.P. pp. 771776.

Reis, C. A., N. A. P. Silva and A. Messaoudi (2004). Determinação de Overshoot e Undershoot em Sistemas de Controle Lineares Estáveis e Contínuos de Terceira Ordem Com pólos e Zeros Reais. Anais do DINCON 2004, $3^{O}$ Congresso Temático de Aplicações de Dinâmica e Controle da SBMAC, Ilha Solteira, S.P., pp. 382 - 390.

Reis, C. A., A. Messaoudi and N. A. P. Silva, (2004-a). Análise de Extremos em Sistemas de Controle Lineares Contínuos de Terceira Ordem Com Pólos e Zeros Reais Estáveis. III Congresso Nacional de Engenharia Mecânica (CONEM 2004), Belém, PA. 
Silva, N. A. P. and C. A. Reis (2001), On The Definition of Type $\mathrm{R}_{\mathrm{u}}$ Undershoot in The Step Response. Anais da $1^{a}$ Escola Brasileira de Aplicações em Dinâmica e Controle, São Carlos, S.P. pp.170-175.

Teixeira, M. C. M., N. A. P. Silva, C. A. Reis and E. Assunção (2002). Projeto de Sistemas de Controle Com Atualização das Condições Iniciais no Controlador. XIV Congresso Brasileiro de Automática, Natal, RN. pp. 1341- 1348.

Teixeira, M. C. M., N. A. P. Silva, C. A. Reis and E. Assunção (2002-a). Projeto de Reguladores Quadráticos Ótimos Com Atualização das Condições Iniciais no Controlador. I Congresso Temático de Dinâmica, Controle e Aplicações. (DINCOM 2002), São José do Rio Preto, S.P., pp. 993-998.

\section{APÊNDICE}

PROVA DO LEMA 01: Por hipótese, $y(0)=0, y^{\prime}(0)=0$, $\ldots, y^{(n-1)}(0)=k$ sendo que:

$$
k=(-1)^{n+1} \frac{\prod_{i=1}^{n} \lambda_{i}}{z}
$$

Agora, basta observar que:

sendo:

$$
c_{1}=\frac{\Delta_{1}}{\Delta}
$$

$$
\begin{aligned}
\Delta_{1} & =\left|\begin{array}{cccc}
-1 & 1 & \cdots & 1 \\
0 & \lambda_{2} & \cdots & \lambda_{n} \\
\cdots \cdots \cdots \cdots \cdots & \ldots \cdots \cdots \\
k & \lambda_{2}^{n-1} & \cdots & \lambda_{n}^{n-1}
\end{array}\right| \mathrm{e} \\
\Delta & =\left|\begin{array}{cccc}
1 & 1 & \cdots & 1 \\
\lambda_{1} & \lambda_{2} & \cdots & \lambda_{n} \\
\cdots \cdots \cdots \cdots \cdots \cdots \\
\lambda_{1}^{n-1} & \lambda_{2}^{n-1} & \cdots & \lambda_{n}^{n-1}
\end{array}\right| .
\end{aligned}
$$

Como $\Delta$ é um determinante de Vandermonde, tem-se que:

$$
\Delta=(-1)^{\frac{n(n-1)}{2}} \prod_{1 \leq i<j \leq n}\left(\lambda_{j}-\lambda_{i}\right) .
$$

Por outro lado:

$$
\Delta_{1}=-\lambda_{2} \ldots \lambda_{n} V\left(\lambda_{2}, \ldots, \lambda_{n}\right)+(-1)^{n+1} k V\left(\lambda_{2}, \ldots, \lambda_{n}\right)
$$
na qual

$$
\begin{aligned}
& V\left(\lambda_{2}, \ldots, \lambda_{n}\right)=\left|\begin{array}{cccc}
1 & 1 & \cdots & 1 \\
\lambda_{2} & \lambda_{3} & \cdots & \lambda_{n} \\
\ldots \ldots \ldots \ldots \ldots \ldots \ldots . \\
\lambda_{2}^{n-2} & \lambda_{3}^{n-2} & \ldots & \lambda_{n}^{n-2}
\end{array}\right| \\
& =(-1)^{\frac{n(n-1)}{2}} \prod_{2 \leq i<j \leq n}\left(\lambda_{j}-\lambda_{i}\right) .
\end{aligned}
$$

Logo,

$$
c_{1}=\frac{\lambda_{2} \cdots \lambda_{n}}{z}\left(\lambda_{1}-z\right) \frac{\prod_{2 \leq i<j \leq n}\left(\lambda_{j}-\lambda_{i}\right)}{\prod_{1 \leq i<j \leq n}\left(\lambda_{j}-\lambda_{i}\right)}
$$

o que prova (5) - (i). De maneira análoga mostra-se que:

$$
c_{2}=-\frac{\lambda_{1} \lambda_{3} \cdots \lambda_{n}}{z}\left(\lambda_{3}-z\right) \frac{\prod_{\substack{1 \leq i<j \leq n \\ i, j \neq 3}}\left(\lambda_{j}-\lambda_{i}\right)}{\prod_{\substack{1 \leq i<j \leq n\\}}\left(\lambda_{j}-\lambda_{i}\right)},
$$

$$
c_{k}=(-1)^{k+1} \frac{\prod_{i=1}^{n} \lambda_{i}}{\lambda_{k} z}\left(\lambda_{k}-z\right) \frac{\prod_{\substack{1 \leq i<j \leq n \\ i, j \neq k}}\left(\lambda_{j}-\lambda_{i}\right)}{\prod_{\substack{1 \leq i<j \leq n \\ 1}}\left(\lambda_{j}-\lambda_{i}\right)}, \quad \forall 3 \leq \mathrm{k} \leq \mathrm{n} .
$$

o que prova o resultado desejado em (6) - (ii).

PROVA DO COROLÁRIO 01: Suponha que $\lambda_{n}<z<0$. Então, de (5), tem-se que:

$$
c_{l}^{(1)}=c_{1} \lambda_{1}=\frac{\lambda_{1} \lambda_{2} \cdots \lambda_{n}}{z}\left(\lambda_{1}-z\right) \frac{1}{\left(\lambda_{2}-\lambda_{1}\right)\left(\lambda_{3}-\lambda_{1}\right) \ldots\left(\lambda_{n}-\lambda_{1}\right)}
$$

Portanto,

$$
\operatorname{sinal}\left(\lambda_{1} c_{1}\right)=(-1)^{n} \sin a l\left(\frac{\lambda_{1}-z}{z}\right)=(-1)^{n} .
$$

o que prova o resultado desejado. Agora, de (5) e (6), temse que

$$
\left(\frac{c_{2}}{c_{1}}\right)<0
$$


Como $c_{1}<0$, para $n$ par, segue que $\mathrm{c}_{2}>0$. De forma análoga, tem-se que

$$
\left(\frac{c_{k}}{c_{k-1}}\right)<0
$$

o que prova o resultado desejado. A prova de (b) é similar.

PROVA DO LEMA 02: De (10), (11), (12) e (13), depreende-se que:

$$
f_{n-2}(t)=1+\left(d_{n-1}^{(n-2)} e^{\lambda_{n-1}^{(n-2)} t}+d_{n}^{(n-2)} e^{\lambda_{n}^{(n-2)} t}\right) .
$$

Daí existe um número real $t_{o}^{*}$ tal que:

$$
y^{\prime}\left(t_{o}^{*}\right)=0 \Leftrightarrow-\frac{d_{n-1}^{(n-2)} \lambda_{n-1}^{(n-2)}}{d_{n}^{(n-2)} \lambda_{n}^{(n-2)}}>1,
$$

o que prova o Lema 02.

PROVA DO LEMA 03: Pelo Lema 02, existe um número real $t_{o}^{*}$ tal que $y^{\prime}\left(t_{o}^{*}\right)=0$ se e somente se

$$
-\frac{d_{n-1}^{(n-2)} \lambda_{n-1}^{(n-2)}}{d_{n}^{(n-2)} \lambda_{n}^{(n-2)}}>1
$$

Assim, para a análise da desigualdade (15), tem-se que:

$$
\frac{d_{n-1}^{(n-2)}}{d_{n}^{(n-2)}}=\frac{c_{n-1}^{(n-2)}}{c_{n}^{(n-2)}}=\frac{d_{n-1}^{(n-3)} \lambda_{n-1}^{(n-3)}}{d_{n}^{(n-3)} \lambda_{n}^{(n-3)}}=\frac{d_{n-1}^{(n-3)}}{d_{n}^{(n-3)}} \frac{\lambda_{n-1}-\lambda_{n-3}}{\lambda_{n}-\lambda_{n-3}} .
$$

Para todo $1 \leq i \leq n-2$, sejam:

$$
u_{i}=\frac{d_{n-1}^{(i)}}{d_{n}^{(i)}} \quad \text { e } \quad v_{i}=\frac{\lambda_{n-1}-\lambda_{i}}{\lambda_{n}-\lambda_{i}}
$$

Então:

$$
\begin{gathered}
u_{n-2}=u_{n-3} v_{n-3} ; \\
u_{n-3}=u_{n-4} v_{n-4} ; \\
\cdots \cdots . \\
u_{2}=u_{1} v_{1} .
\end{gathered}
$$

Então, de (16) observa-se que:

$$
\begin{aligned}
u_{n-2}= & u_{1} v_{1} v_{2} \cdots v_{n-3} \\
= & \frac{d_{n-1}^{(1)}}{d_{n}^{(1)}} \frac{\prod_{i=1}^{n-3}\left(\lambda_{n-1}-\lambda_{i}\right)}{\prod_{i=1}^{n-3}\left(\lambda_{n}-\lambda_{i}\right)} .
\end{aligned}
$$

Dessa forma, fazendo a análise de (15), conclui-se que:

$$
-\frac{d_{n-1}^{(n-2)} \lambda_{n-1}^{(n-2)}}{d_{n}^{(n-2)} \lambda_{n}^{(n-2)}}=-\frac{d_{n-1}^{(1)}}{d_{n}^{(1)}} \frac{\prod_{i=1}^{n-2}\left(\lambda_{n-1}-\lambda_{i}\right)}{\prod_{i=1}^{n-2}\left(\lambda_{n}-\lambda_{i}\right)}
$$

$$
\frac{d_{n-1}^{(1)}}{d_{n}^{(1)}}=\frac{c_{n-1}^{(1)}}{c_{n}^{(1)}}=\frac{c_{n-1} \lambda_{n-1}}{c_{n} \lambda_{n}}
$$

Portanto, com a substituição de (18) em (17) obtém-se que:

$$
-\frac{d_{n-1}^{(n-2)} \lambda_{n-1}^{(n-2)}}{d_{n}^{(n-2)} \lambda_{n}^{(n-2)}}=-\frac{c_{n-1} \lambda_{n-1}}{c_{n} \lambda_{n}} \frac{\prod_{i=1}^{n-2}\left(\lambda_{n-1}-\lambda_{i}\right)}{\prod_{i=1}^{n-2}\left(\lambda_{n}-\lambda_{i}\right)}
$$

Pelo Lema 02, mostra-se que:

$$
-\frac{c_{n-1}}{c_{n}} \frac{\lambda_{n-1}}{\lambda_{n}}=\frac{\lambda_{n-1}-z}{\lambda_{n}-z} \frac{\prod_{\substack{1 \leq \mathrm{i}<\mathrm{j} \leq \mathrm{n} \\ \mathrm{i}, \mathrm{j} \neq \mathrm{n}-1}}\left(\lambda_{j}-\lambda_{\mathrm{i}}\right)}{\prod_{\substack{1 \leq \mathrm{i}<\mathrm{j} \leq \mathrm{n} \\ \neq \mathrm{n}}}\left(\lambda_{j}-\lambda_{\mathrm{i}}\right)} .
$$

Daí,

$$
-\frac{c_{n-1}}{c_{n}} \frac{\lambda_{n-1}}{\lambda_{n}} \frac{\prod_{i=1}^{n-2}\left(\lambda_{n-1}-\lambda_{i}\right)}{\prod_{i=1}^{n-2}\left(\lambda_{n}-\lambda_{i}\right)}=\frac{\lambda_{n-1}-z}{\lambda_{n}-z}
$$

De (19) e (20), conclui-se que:

$$
-\frac{d_{n-1}^{(n-2)}}{d_{n}^{(n-2)}} \frac{\lambda_{n-1}^{(n-2)}}{\lambda_{n}^{(n-2)}}>1
$$

se e somente se $\lambda_{n}<z$, o que prova o Lema 03 . 